International Journal of Social Science And Human Research

ISSN(print): 2644-0679, ISSN(online): 2644-0695

Volume 05 Issue 02 February 2022

DOI: 10.47191/ijsshr/v5-i2-21, Impact factor-5.586

Page No: 551-561

\title{
Implications of Terrestrial Variations on the utility of Theory of Special Neurodiversity
}

\author{
Rashmi Malhotra ${ }^{1}$, Vinayak Malhotra ${ }^{2}$ \\ ${ }^{1}$ Department of History, Lady Shri Ram College for Women, New Delhi, India \\ ${ }^{2}$ Department of Aerospace Engineering, SRM Institute of Science and Technology, Chennai, TN, India
}

\begin{abstract}
Theory of special neurodiversity mandates the adjacent colonization of neurodiverse people with special needs for upgraded societal reforms and development. As the concept of a nation enfolds diverse geographical variations, the effectiveness of the theory of special neurodiversity can be optimized only if the controlling parameters are broadly understood and resolved in a unified way. For the enhanced socio-economic growth of any nation, with the application of the theory of special neurodiversity, these variables are likely to affect the imperious restructuring which necessitates active research efforts to minimize their unreceptive impact. In view of the futuristic development, a simple youth-oriented sampling research survey was conducted to attain better physical insight about the geographical incongruities and their implications. The study attempts to examine the singular and coupled effect of key controlling geographic parameters viz., culture, climate, coordination, gender. Based on the results obtained, innovative modulations will be accorded in the prevailing RAS-VIN Model of Special Neurodiversity for enhanced socio-economic growth and the contribution of special needs people. The understanding of geographical implications on the theory of special neurodiversity can be effectively applied on the wide range of administrative, institutional, organizational, engineering, humanities, and sciences applications explicitly aiming at upliftment and empowerment of special needs people.
\end{abstract}

KEYWORDS: Special Neurodiversity, geographical variation, socio-economic growth, value system.

\section{INTRODUCTION}

In a vast country like India, diversity is inevitable. We Indians, further take pride in the diversity it represents. From Kashmir to Kanyakumari and Ahmedabad to Shillong, a number of variations in ethnicity, religion, caste, class can be noted along with diverse climatic conditions and terrains. With 22 official languages in the eighth schedule of the Indian constitution, along with 8 ethical identities and 9 major religions, the concept of the 'Indian Culture' is an extremely complex and complicated matter. Through the 'Theory of Special Neurodiversity' and 'RAS-VIN's' model of Special Neurodiversity, the need of the hour for the adjacent colonization of neurodiverse people for the growth of both as an individual and nation was established. In India, with a population reaching up to 1.3 billion, geographical variations may act as a hindrance in the proper utilization of the said model. The reason behind this is diversity. While on the one hand, diversity in the workplace might prove to be beneficial through broader perspective and global reach which we can note through McKinsey's diversity wins data set (figure 1 (a)) where the analysis of 2017 indicated $25 \%$ more possibility of above-average profitability in companies with top quartile for gender diversity on executive teams when compared to those in the fourth quartile and the same was noted in the case of ethnic and cultural diversity where the companies within the top quartile outperformed those in the fourth quartile by $36 \%$ in profitability, it's negative connotations attached to it can't be ignored when this diversity is poorly managed. A few examples like cultural hostilities, unresolved conflicts, poor communication, discrimination, etc. can be taken into account. The focus here is on the socio-economic growth of the nation through proper utilization of the aptitude of people with special needs in the workplace.

When the management of an organization or the workplace becomes too focused on diversity and difference, the corporate objectives and the work productivity might suffer 


\section{Implications of Terrestrial Variations on the utility of Theory of Special Neurodiversity}

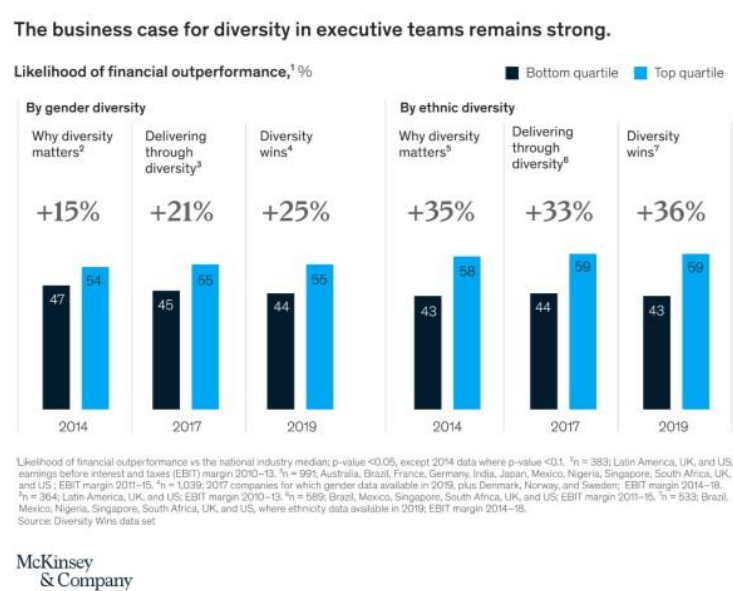

(a)

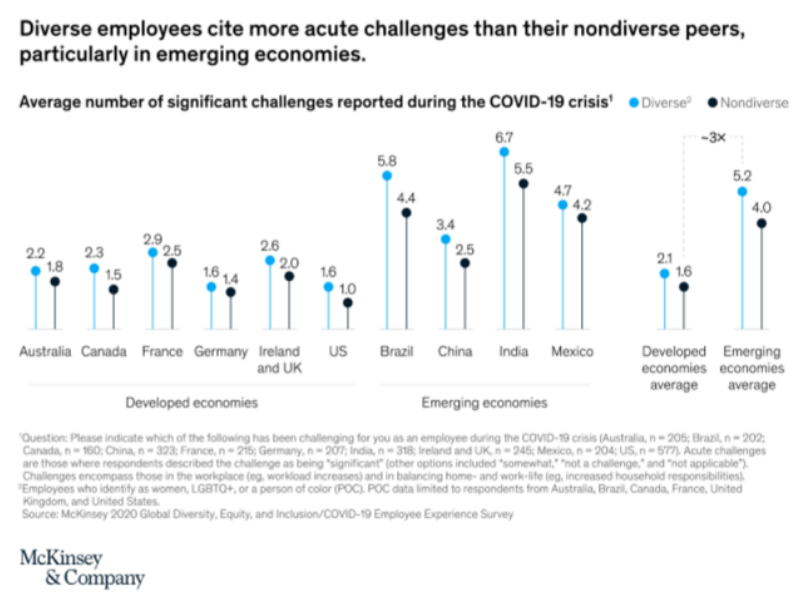

(b)

Fig. 1. Statistical representation of (a) increased work profitability through diversity; (b) challenges faced by the diverse population (*McKinsey \& Company).

While noting the role of geography in the challenges faced in the workspace especially during the

COVID-19 crisis, Mckinsey's 2020 Global (Diversity, Equity and Inclusion) survey (figure 1 (b)) indicated that when compared to their non-diverse peers, diverse employees reported additional challenges in every country. In the context of India, diverse employees experienced 6.7 acute challenges on average while non-diverse employees experienced 5.5 challenges for the same. Furthermore, when taking note of the demographic variations in the people with special needs, it was noted that in a country like India where around $2.2 \%$ i.e., 2.6 crores of the population comprise people with special needs, nearly $80 \%$ are out of the labor force. Furthermore, people with special needs have a mere LFPR of $23 \%$ as a whole when compared to 50\% LFPR in all India. While reservation and PSUs might play a significant role in reducing these disparities, a proper representation of people with special needs is still absent. Accessibility and discrimination contribute to being some of the main reason behind people with special needs being unemployed or engaging in more freelance work or being self-employed. While $22.8 \%$ of all the Indian population are salaried workers, only $15 \%$ of people with special needs hold regular jobs. 59.7\% of the special need population still optimate for selfemployment (see figure 2).

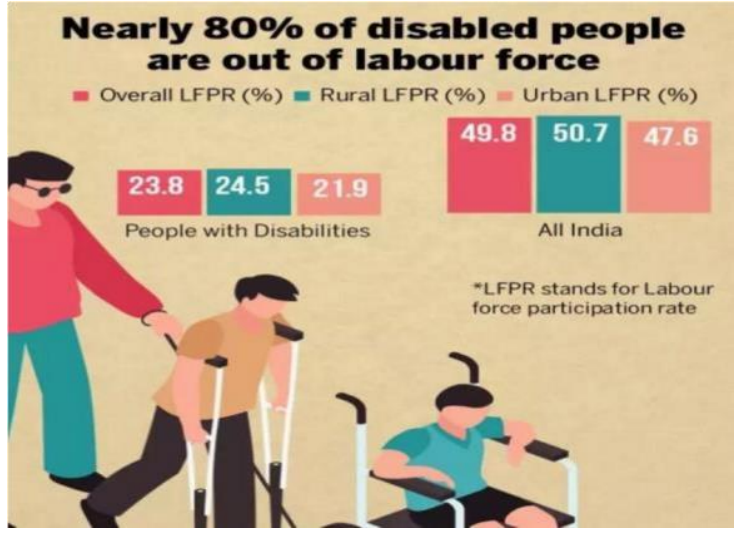

(a)

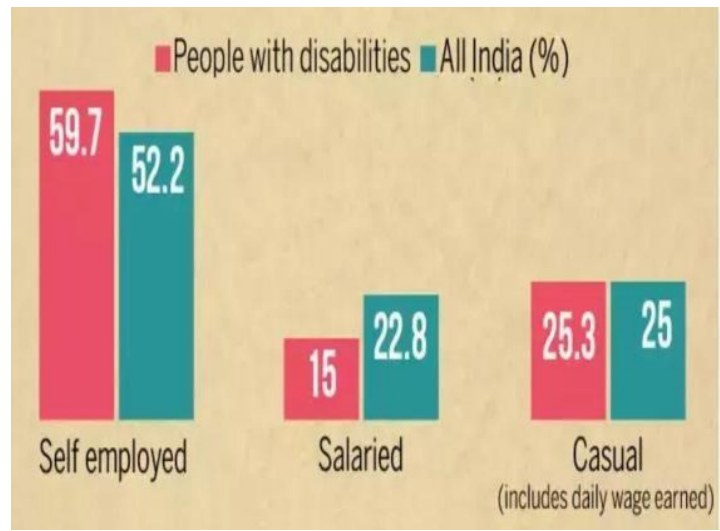

(b)

Fig. 2. Statistical representation of (a) LFPR (b) employment type of people with special needs.

This situation is not just prominent in India alone. Even in a developed country like the U.S. (figure 3) the difference/gap between the disabled and non-disabled employed population is quite large.

Taking note of the 2012 employment-population ratio, while $63.9 \%$ non-disabled population was employed, only $17.8 \%$ disabled population was employed. 


\section{Implications of Terrestrial Variations on the utility of Theory of Special Neurodiversity}

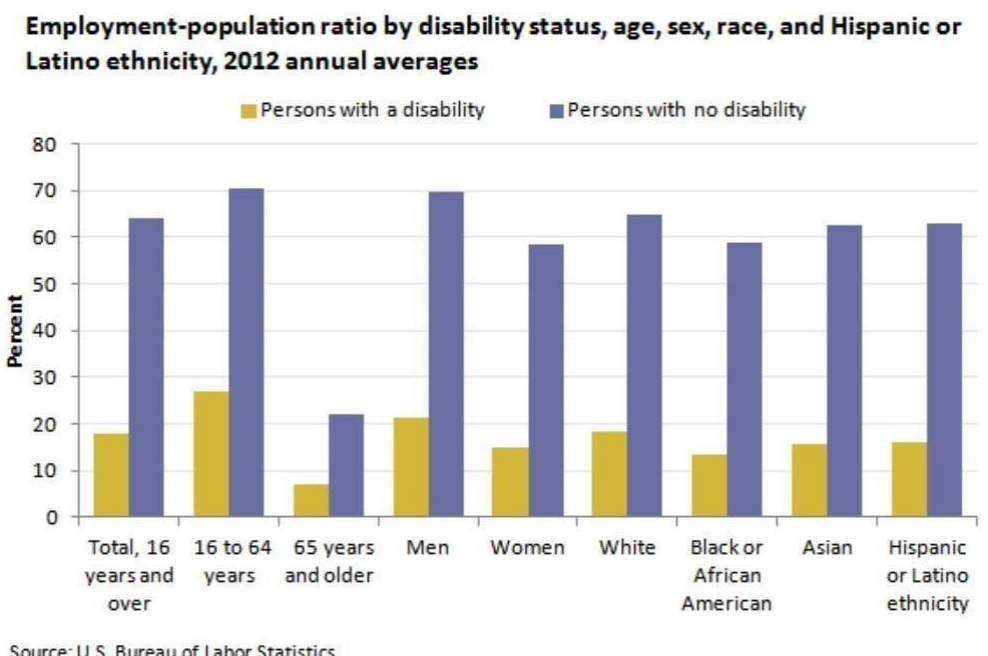

Fig. 3. Statistical variation of employment-population ratio in the U.S. (2012) (* google.com).

A new system keeping the range of the DE\&I (Diversity, Equity \& Inclusion) high in any organization will enable it to drive the impact of change through action. The issue of accessibility needs to be tackled, external differences not pertaining to work needs to be put aside. Rather than making an individual feel isolated because of their disability, the focus should be on their work proficiency. Only through this the maximum optimization of 'Theory of Special Neurodiversity' is achievable. And for this to be possible, the role of the 3 key geographical parameters needs to be utilized to their significant quantity. We can achieve a proper outcome by only focusing on issues like accessibility, cultural differences, barriers, etc. Proper coordination between all these will help us achieve it. When the issue of accessibility in case of people with special needs is tackled upon, the work proficiency is bound to increase; when the focus of the organization is on how an individual can make a significant contribution to the organization and the nation along with utilizing their talents rather than the limitation that may occur because of the disability of an individual, their mental health is bound to improve, and better mental health result into more productivity and thus, accelerating the socio-economic growth of the nation.

Following the classical work of Hall [1] on the intertwined geographies of social addition for people with learning disabilities. Appreciable work had been carried out which primarily bestowed with the development of an understanding of the physical phenomena associated with the geographical effect on systems comprising working special needs people. Park et. al., [2] explored and reviewed the intensifying literature on disability, charting the process of the significant studies in the field. The work primarily focussed on the physical disability followed by the issues around mental health and intellectual disability. The study evaluated and resolved the implication of topical work on disability within human geography as a whole and suggested possible links to the growing literature on rights, social justice, and ethics. Lemon and Lemon [3] reported that the potential of intellectual special needs people can noticeably improve and imply in managing their households and the workplaces. The study affirmed that the success of such an economic model can happen with community support viz., families, social workers, and from non-profit groups and public services such as social housing and public transit. The leading movements among social sector institutions of people with intellectual disabilities have produced models that focus on deficiencies, individualization, and service. The work stated that through cooperative structures and entrepreneurial activity, projects in community economic development can replace these models with an emphasis on capacities, collectivization, and care.

Imrie and Edwards [4] highlighted the interest of geographers in the subject of disability confined beyond the discipline. The study signified that geographical necessity about space, place, and disability has multiplied. The work in principle assessed and outlined the consequence of theoretical and methodological developments in the sub-discipline. The result represented the ways in which studies of geography and disability are likely to evolve. Singal [5] focused on the potential for social transformation of the youth with disabilities. The investigation reflected that, special needs people in the 12-24 years age form a significant minority whereas, the knowledge about their experiences, how others around them make sense of their lives and perceive their transition is not much known. The study adapted the transition framework proposed by the World Bank's World Development Report 2007 to inspect chances for young people with disabilities in the areas of learning, work, citizenship, and their prospects in a time of optimism. Appunni and Deshpande [6] reiterated that the special needs people are keen to overcome the social and economic barriers. The work attempted to study the work participation rates among incapacitates in India by using Census of India 2001 data. It was observed that out of 1.03 billion populations about 2.1 percent of the population is of disable persons and of which 75 percent survives in rural areas. The exploration highlighted that the work participation among disabilities in India is about $35 \%$ males which is double than the females. Moreover, in general the work participation is higher than that among disables. The study asserted the need to increase the work participation among disables especially among females. Shenoy [7] worked on the key challenges and 


\section{Implications of Terrestrial Variations on the utility of Theory of Special Neurodiversity}

issues faced by special needs people in Indian labour markets. The study detailed an outline of the major organizations and institutions acting for persons with disability in India and to recognize tactical prospects to improve and expand persons with disability contribution in Indian labor markets.

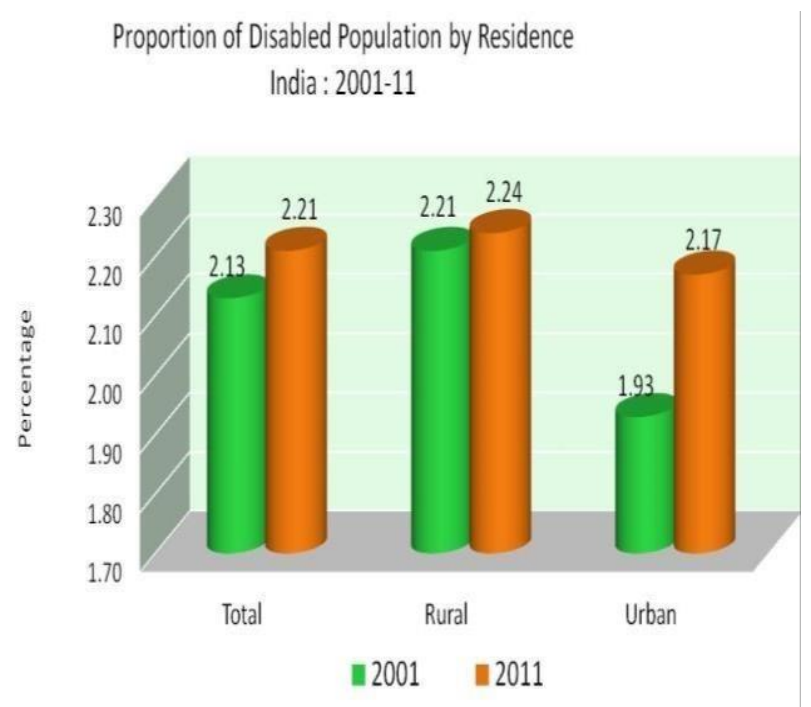

(a)

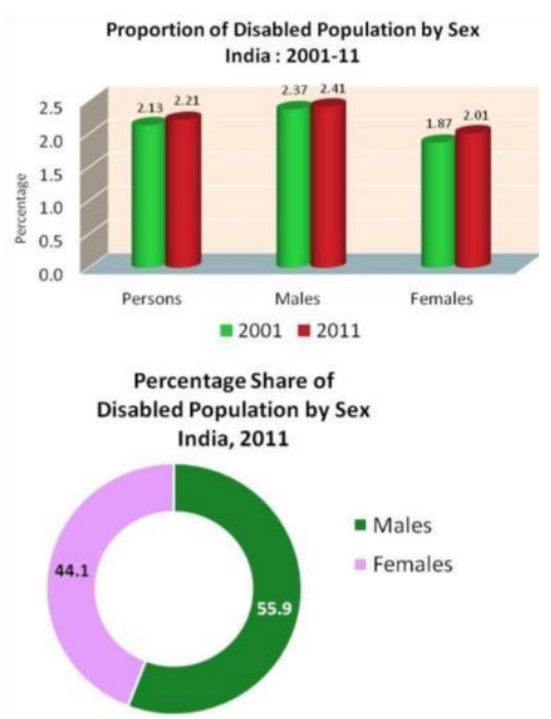

(b)

Fig. 4. Disabled population by (a) residence and (b) sex in India: Census 2001-2011(*google.com).

The results from the study recommended key interventions that could be pursued by the ILO in India. In India, according to the Census 2011(fig.4), disabled persons constituted $2.21 \%$ of the total population. In terms of geographical variation, the census revealed that, among all State/UTs, the percentage of special need people to the total population is highest in Sikkim (2.98\%), followed by Odisha (2.96\%), Jammu \& Kashmir (2.88\%), Andhra Pradesh (2.68\%), and Maharashtra (2.64\%). Whereas, the proportion of disabled persons to the total population is lowest in Daman \& Diu (0.9\%), Dadra \& Nagar Haveli $(0.96 \%)$, Mizoram (1.38\%), Delhi (1.4\%) and Chandigarh (1.4\%). Nearly $50 \%$ of the disabled persons belonged to one of the five States namely Uttar Pradesh (15.5\%), Maharashtra (11.05\%), Bihar (8.69\%), Andhra Pradesh (8.45\%), and West Bengal (7.52\%).

At all India level, $36.34 \%$ of the total disabled population was testified as workers. Nagaland $(51.92 \%)$ has the highest share of special need workers followed by Sikkim (49.04\%) and Arunachal Pradesh (44.69\%). The proportion of disabled workers to the conforming total disabled persons was reported as lowest in Lakshadweep (19.88\%), followed by Kerala (23.59\%) and NCT of Delhi (27.92\%). The highest number of disabled workers were noted to be from Uttar Pradesh (14.84\%), followed by Maharashtra $(12.81 \%)$, which have also the highest number of disabled persons. In terms of literacy, $54.52 \%$ of the disabled persons are literates in India. The highest literacy rate among disabled persons was reported in Kerala $(70.79 \%)$ followed by Goa (70.31\%) whereas, the lowest literacy rate among disabled persons was testified in Arunachal Pradesh (38.75\%) followed by Rajasthan (40.16\%). Nationally, $8.53 \%$ of the disabled literates were reported as having educational qualification of graduate and above with the highest share of graduates in the disabled literate population in Chandigarh (19.68\%) followed by Delhi (17.43\%) and the lowest share in Lakshadweep (2\%) followed by Meghalaya (3\%). Bihar has the highest number of persons with disability in speech while under the various categories of disability like disability in seeing, in hearing, in movement, mental retardation, mental illness, any other and multiple disability, Uttar Pradesh has the highest number. The highest percentage of disabled children (5-19 years) presently attending educational institution were reported from Goa \& Kerala (73\%) followed by Maharashtra and Lakshadweep (70\%) however, Nagaland (39\%) followed by Assam (35\%) reported the maximum percentage of disabled children who never attended educational institution. The data clearly validated the geographical variation of special needs people under working category and undecided addressal of the issues related to their upliftment. Kumar et. al., [8] studied disability as an important public health problem which will increase in future because of upsurge in non-communicable diseases and change in age structure. The work reiterated that recuperation measures should be targeted according to the requirements of the disabled with community participation. The study analyzed data from Medline to understand various issues and challenges related to disability and rehabilitation services and stressed to support health care and service delivery to disabled in the community. The results highlighted suitable intrusion strategies and their implementation to the present context. Jindal and Chari [9] compiled the employment situation of special needs people in India in retrospect to the disability act (1995). The study primarily discussed the growth of the employment scenario for people with disabilities owing to the Act in 1995 and the modulations needed. The case study in collaboration with the census 2011 reported that of the total population only $26 \%$ constitute the working population of which $78 \%$ are males. It was reported that there is greater unemployment among women with disabilities. The observation accorded that in spite of the huge resource pool, employment issues of disable people are extremely low on the agenda. 


\section{Implications of Terrestrial Variations on the utility of Theory of Special Neurodiversity}

Owing to the disability act (1995), selected prospects opened up particularly in the area of education and employment, however, more work needs to be done for the country to look at persons with disabilities as contributing human resources. Limaye [10] assessed and stated that children with disabilities are amongst the most disadvantaged in terms of access to schooling and completion of elementary education, as their needs are not met effectively. The study explored challenges in the education system with respect to issues of education quality and drop-out rates of students with disabilities. Results highlighted that factors that principally influence the accessibility of education for disabled children includes, perceptions of parents, the general societal attitude, government officials, school staff and infrastructure, poverty, lack of acceptance, lack of interest, gender discrimination, lack of awareness, poor physical access, availability of various support systems, and the government strategies focusing on the education of disabled children in definite. Mehta et. al., [11] carried out a study to understand the disabled-friendly work environment of the Indian organizations by implementing the quantitative methodology to understand the standing of disabled workforce in India.

The exploration recommended that various practices and policies should be added in the organizations to create a disabled-friendly environment. Furthermore, the study suggests that Indian organizations need amendment in engaging disabled employees at workplace ways through which comprehensive work culture can be promoted. At organization level, positions should be offered to disabled at all levels rather than at lower levels. It is likely to contribute to an incipient framework on employment integration of disable people and the role of employers. Seilan [12] conducted a study to reiterate that disabled persons were the most omitted ones in the progress of the country. Considering the importance of geographical location, the study corroborated with the fact that $76 \%$ of disabled persons were from rural areas and only $24 \%$ were from urban areas and $59 \%$ were males and only $41 \%$ were females. Results from the exploration detailed that the government and non-government foundations need to range more support to the disabled persons in addition to wide-ranging and unbiased provision for inclusive education and employment. In recently, Malhotra and Malhotra [13] offered solution to the widely spread ambiguity in the special needs working class in the form of 'Theory of Special Neurodiversity' and 'RAS-VIN's model'. The work ascertained various complications faced by people with special needs and attempts to provide a closed form solution for the same. Based on the results, an innovative model for working with special needs people was proposed which was engrossed in making neurodiverse special needs working people to stay together for enhanced professional contribution of special needs people. Among all the appreciable work carried out, one of the aspects which is yet to be comprehensively established is the role of geographical variation as a key controlling parameter in working disabled persons. Present study is motivated by the need for mental and technological advancement of working special needs people coupled with uncomplicatedness in terms of time, energy and money. The investigation primarily focuses on optimizing the output through application of 'Theory of special Neurodiversity', under varying operating conditions leading to a proficient model for working with special needs people. The study is motivated by the need. The specific objectives of the study are:

1) To identify the effect of geographical variation on prospects of working special needs people coupled with application of 'Theory of special neurodiversity'.

2) To develop appropriate correlations to understand the governing dynamics of the geographical variations and its implications on the 'Theory of special neurodiversity' application.

3) To understand the role of key controlling parameters.

\section{SURVEY RESEARCH AND SOLUTION METHODOLOGY}

To accomplish the specific objectives, the research method of surveying was adapted. India has more than 50\% of its population below the age of 25 and more than $65 \%$ below the age of 35. To explore the geographical variations of the 'Theory of Special Neurodiversity', a youth-oriented survey was conducted within a controlled social atmosphere. Selected number of participants with age criteria from 15-35 years old were chosen. The diversity of the participants was maintained in the form of students of highergrade sections, undergraduate students, and academic professors. The survey was sent to participants through text/email with a brief introduction to the objectives of the survey. The survey form comprised of eight quintessential questions as understated:

1) Can we accept the 'Theory of Special Neurodiversity' as a closed-form solution for enhancing the productivity and living standards of people with special needs?

2) Do you think geographical variation can alter the utilization of the 'Theory of special neurodiversity'?

3) In view of geographical modulation, the factors which are most likely to affect the utility of the 'Theory of Special Neurodiversity' viz., culture, climate, coordination or none of them.

4) In view of cultural geographical variation, do you think the effects of 'Theory of Special Neurodiversity' can vary with gender?

5) In view of cultural geographical variation, do you think the effects of 'Theory of Special Neurodiversity' can vary with religion/caste/race?

6) If climate geographical variation affects the utility of 'Theory of Special Neurodiversity' then, under which terrain its utility can be easily optimized viz., rains, mountains, hot weather, plains. 


\section{Implications of Terrestrial Variations on the utility of Theory of Special Neurodiversity}

7) In view of coordination geographical diversity, do you think the present-day lifestyle can affect the application of 'Theory of Special Neurodiversity'?

8) For special needs people with geographical diversity, do you think age can alter the effects of 'Theory of Special Neurodiversity'?

Based on the response(s) useful insight was drawn leading to the enhanced understanding of 'Theory of special neurodiversity' for broad range of applications.

\section{RESULT AND DISCUSSION}

In the preceding work [13], 'Theory of Special Neurodiversity' was understood along with the need for neurodiverse colonization and the reason(s) behind it. Prior to the main survey, the fundamental understanding of the primary issue addressed with 'Theory of Special Neurodiversity' was questioned for testing, and validation. It was surveyed whether the 'Theory of Special Neurodiversity' can be considered in its original form as a closed solution for enhancing productivity and living standards of special needs people. Figure 5 highlights that $\mathbf{4 4 . 5 9 \%}$ of the youth firmly believes it does in comparison to fractional $\mathbf{1 8 . 9 1 \%}$ who believes it won't work while $\mathbf{3 5 . 1 \%}$ were uncertain about its validity. In this work, the factors affecting optimal utility of 'Theory of Special Neurodiversity' was searched. As a vast and diverse land like India is principally affected by the geography in its operational and functional form.

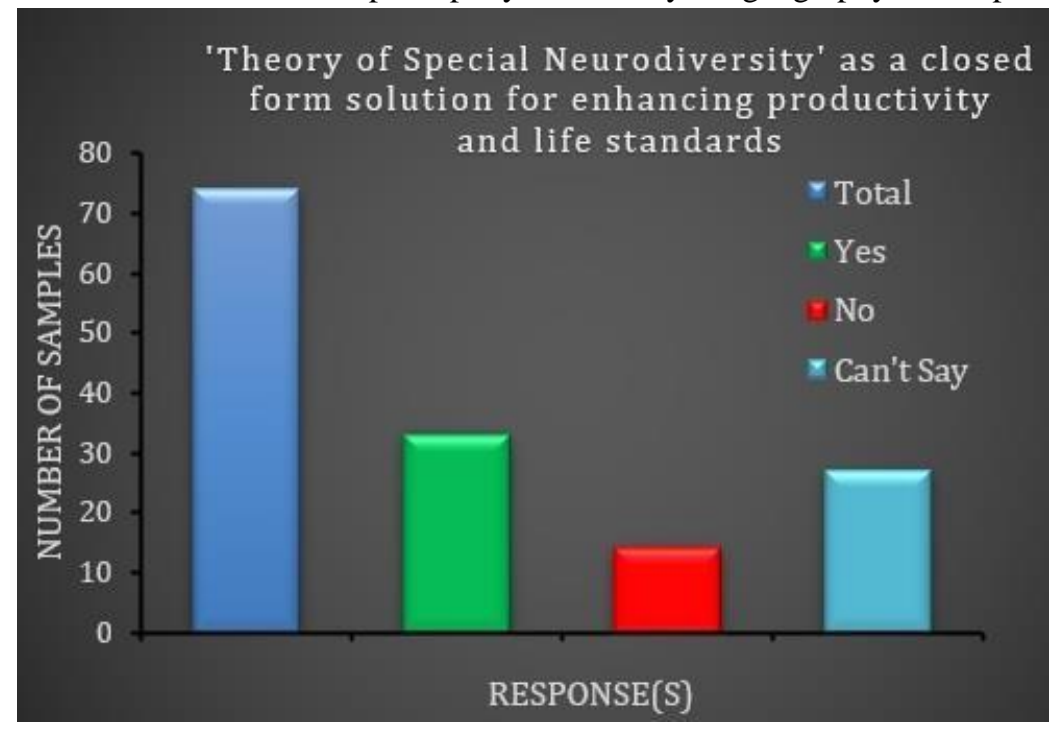

Fig. 5. Statistical variation on the acceptance of 'Theory of Special Neurodiversity' as a closed-form solution.

Present work attempts to venture into the territory of geography and how it can affect utility of 'Theory of Special Neurodiversity', primarily in the form of the 3-C i.e., climate, culture, and coordination laterally with taking note of both internal and external factors. The investigation comprises parametric variation both from a singular and coupled perspective. The results highlight the mentality/mindset of the present youth. Firstly, it was questioned whether the utility of 'Theory of Special Neurodiversity' will absolutely get modulated with a geographical variation.

Figure 6 shows the result of the variation of opinion regarding the same. When $\mathbf{6 8 . 9 1 \%}$ of the sampling strength purports it, $\mathbf{8 . 1 1 \%}$ percent strongly denies it. while $\mathbf{2 2 \%}$ remain unsure about their answer.

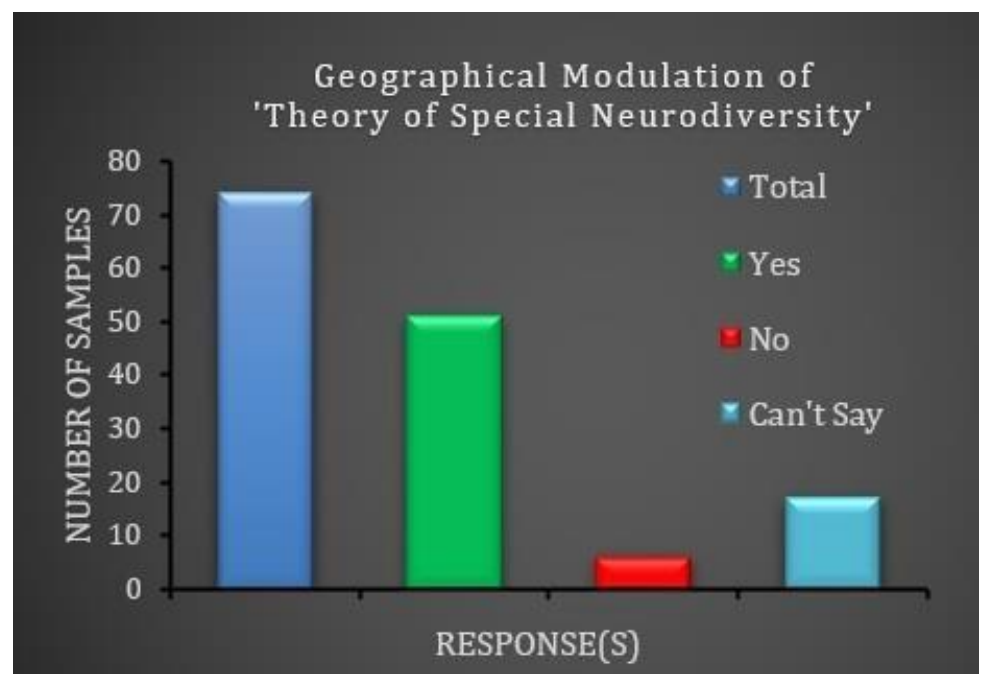

Fig. 6. Statistical variation of the effect of geography on the utility of 'Theory of Special Neurodiversity'. 


\section{Implications of Terrestrial Variations on the utility of Theory of Special Neurodiversity}

Next, the role of three primary factors being culture, climate, and coordination was explored in affecting the utilization of the 'Theory of Special Neurodiversity'. As the survey conducted is youth-oriented interestingly, $\mathbf{5 9 . 4 5 \%}$ believe culture variation with geography will modulate 'Theory of Special Neurodiversity'. However, $\mathbf{1 4 . 8 6 \%}$ states climate and $\mathbf{1 6 . 2 1 \%}$ label coordination variation with geography largely will modulate the utility of 'Theory of Special Neurodiversity' as shown in figure 7. It was also pointed out that socio-economic privileges might play a significant role in the same.

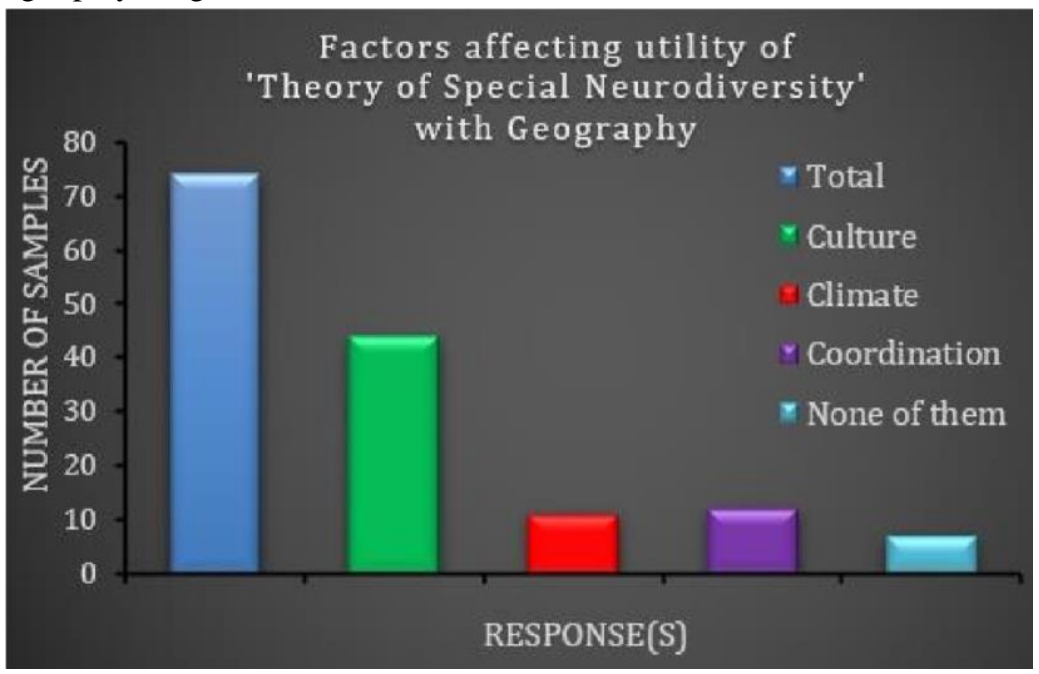

Fig. 7. Statistical variation of the three key parameters viz., climate, culture, and coordination.

Further, the utilization of the 'Theory of Special Neurodiversity' for gender variation was investigated. Figure 8 indicates that $\mathbf{7 5 . 6 7 \%}$ of the present youth believe that results of 'Theory of Special Neurodiversity' will largely vary for different genders, whereas $\mathbf{8 . 1 1 \%}$ of the strength believes it won't. 16.21\% of the participants had no opinion on the same. The reason for the variation for the outcome can be attributed to the diversity of mindset, upbringing, expectations attached to different genders along with the stereotypes attached to the same.

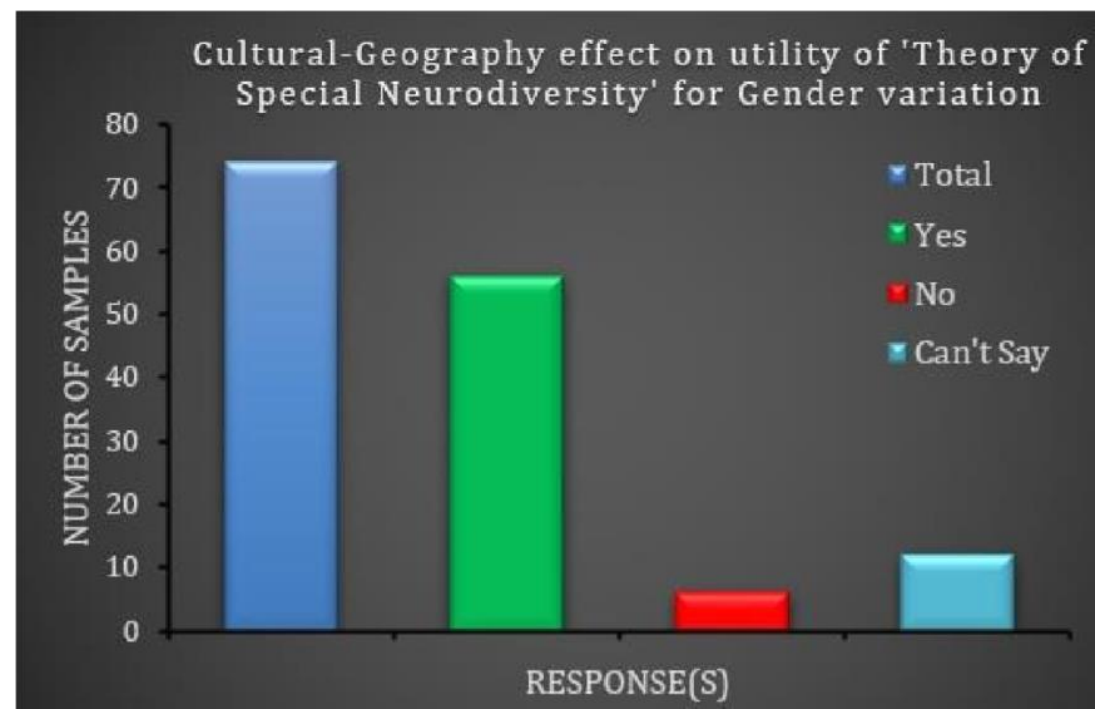

Fig. 8. Statistical variation of the effect of cultural geography on 'Theory of Special Neurodiversity' and its implications on gender variation.

Following which, the role of the cultural-geography especially viz., caste, class, and religion was explored. As shown in figure 9, $\mathbf{6 4 . 8 6 \%}$ of the participants advocates that utility of 'Theory of Special Neurodiversity' will be hampered with geographical diversity whereas $\mathbf{1 6 . 2 1 \%}$ indicates insensitivity to the same while $\mathbf{1 8 . 9 1 \%}$ provides no insight for the same. It goes without saying that people belonging to different geographical locations have diversity in caste, class, religion, etc. This diversity creates a series of different attitudes. So, it's by no doubt that these differences will result in different levels of utilization of 'Theory of Special Neurodiversity' at different locations. 


\section{Implications of Terrestrial Variations on the utility of Theory of Special Neurodiversity}

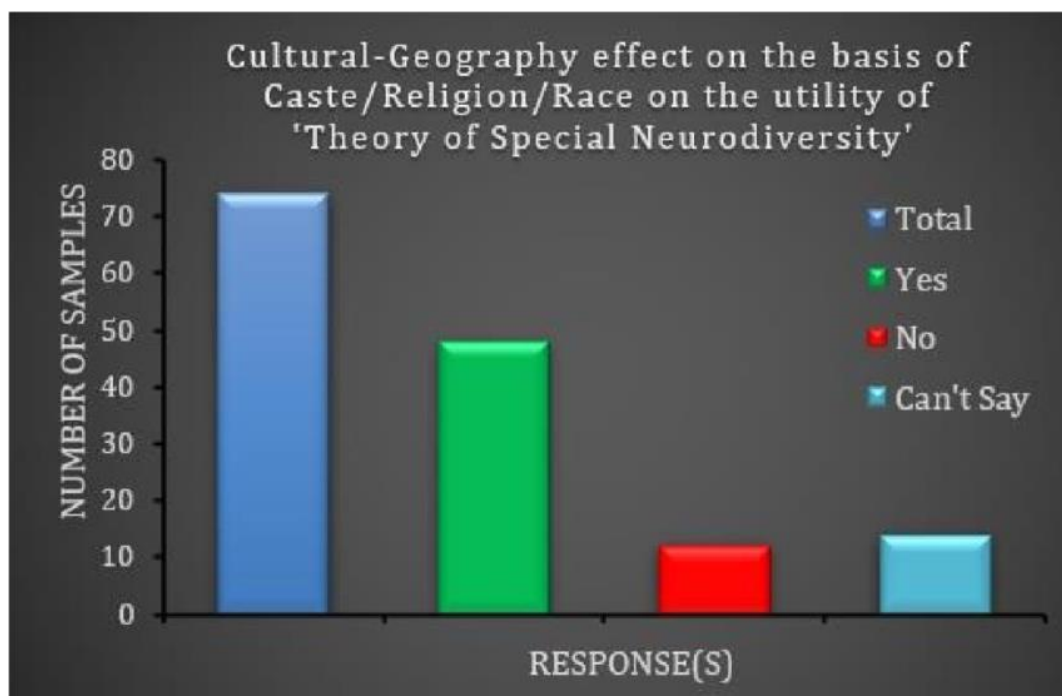

Fig. 9. Statistical variation on the impact of cultural variation (caste/ religion/ race) on 'Theory of Special Neurodiversity'.

While for National Growth, 'Theory of Special Neurodiversity' must be applied throughout and that brings in varying climates with different terrains. Figure 10 indicates that $\mathbf{6 7 . 5 6 \%}$ of the participants retained the mindset of maximizing the utility of 'Theory of Special Neurodiversity' at plains whereas $\mathbf{1 0 . 8 1 \%}$ of the participants resolved to mountains, rains, and hot climate-induced terrains. Diversity in climates brings us to the fact that people from different terrains might have further differences in their level of adaptability. Subsequently, one of the most important controlling factors viz., effectiveness of mutual coordination, for the utilization of 'Theory of Special Neurodiversity' was explored.

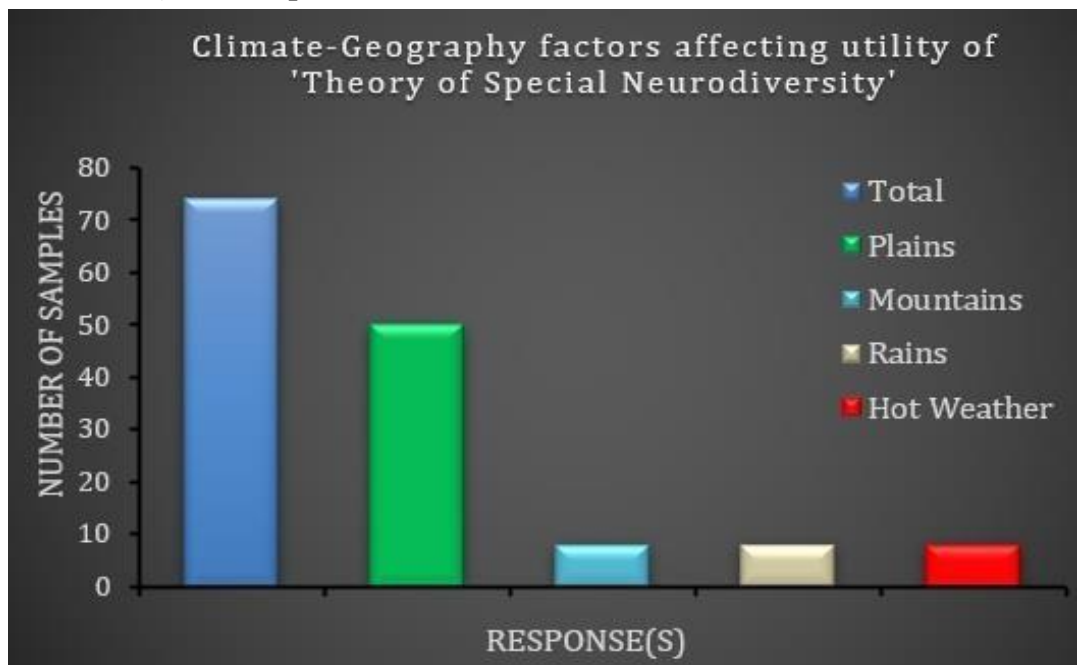

Fig. 10. Statistical variation of the effect of climate geography on 'Theory of Special Neurodiversity'.

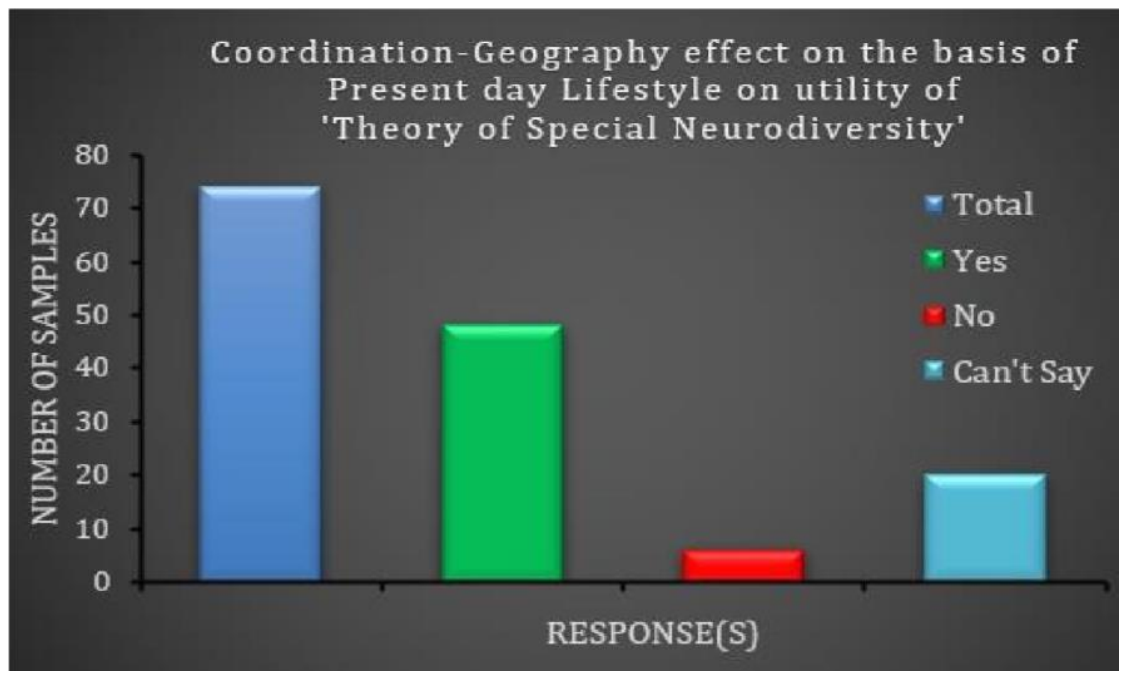

Fig. 11. Statistical variation of the effect coordination-geography on 'Theory of Special Neurodiversity' 


\section{Implications of Terrestrial Variations on the utility of Theory of Special Neurodiversity}

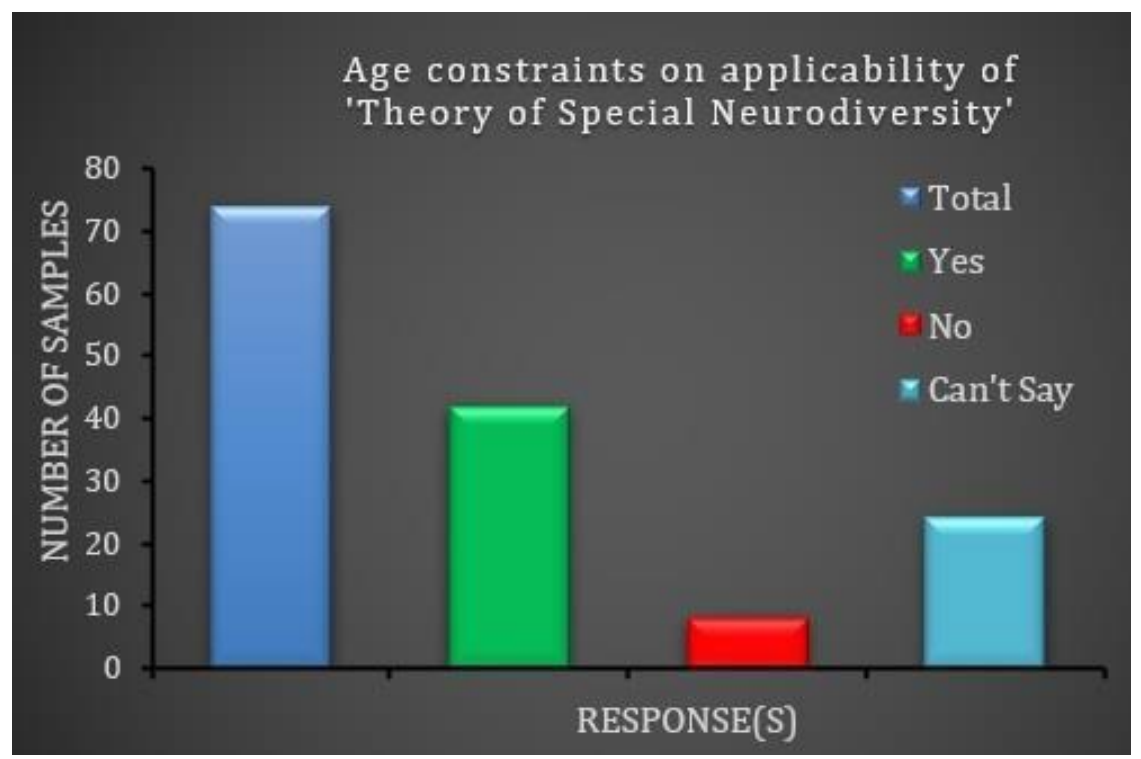

Fig. 12. Statistical variation on the impact age variation on 'Theory of Special Neurodiversity'

It was questioned whether, in present times, coordination can get affected with varying geography. As noted in figure $11, \mathbf{6 4 . 8 6 \%}$ of participants agree that geographical variation will alter mutual coordination whereas, $\mathbf{2 7 . 0 2 \%}$ represent neutrality and marginal $\mathbf{8 . 1 1 \%}$ believe that the utility of 'Theory of Special Neurodiversity' with coordination will not get modulated with varying geography. At last, the role of age variations in different geographical locations was enquired about. Figure 12 conveys that $\mathbf{5 6 . 7 5 \%}$ of youth believes that output of 'Theory of Special Neurodiversity' will vary with age in comparison to marginal $\mathbf{1 0 . 8 1 \%}$ who believe age won't become a constraint while $\mathbf{3 2 . 4 \%}$ chose not to answer this question. Different parts of the community belonging to different sections have different morals and values. While it might be easy to develop a change of mindset in the present-day youth, it cannot be same for the older generation. A reason behind this can be the fact that while the mindset of the older generation is primarily focused on culture, the new generation provided more importance to coordination as compared to the other.

From the above results, one can draw the insight that each of these three parameters i.e., the 3-C (culture, climate, and coordination) play an undoubtedly significant role in the utilization of 'Theory of Special Neurodiversity' and the proper optimization of 'Theory of Special Neurodiversity' through singularity i.e., by focusing on one single factor can't be achieve. Rather, what is needed is a solution through a coupled perspective. This brings us to a new proposed model as a solution entitled 'Dynamic Coordination Equilibrium Model'. The main feature of this model indicates that in any working system considering the neurodiversity of special need people across any geographical location, to obtain maximum benefits of 'Theory of Special Neurodiversity', the equilibrium of the dynamic coordination should be maintained in concurrence with neurodiversity.

To elucidate it further, one can understand the fact that culture and climate are two of the biggest issues in coherence with geography. Both these factors can be proven as a hindrance to the maximum utilization of the 'Theory of Special Neurodiversity'. The fact that people belonging to different cultures have different mindsets pertaining especially to caste, class, race, and religion along with different reactions to the same situations is quite obvious. The difference in mindset sometimes proves to be a significant problem because of the conflicting views and thus, leading to chaos and conflicts. Furthermore, the climate is always changing with geography. Different climatic conditions in different terrains have a different impact on an individual. We can take seasonal depression as one of the examples for the same. Therefore, change in this system is dynamic by nature. To minimize the hindrance caused by these two factors and prevent them from affecting work productivity, what is needed is proper coordination. For it, the focus needs to be work or productivity rather than the differences and dynamic coordination proves to be a significant solution. To put it in simple words, irrespective of your culture or gender or climate change or difference because of geography, equilibrium of the coordination can still help you get maximum benefits from 'Theory of Special Neurodiversity'.

It is important to note that the dynamic coordination is different from normal coordination. While normal coordination might help two in working together at an average level, dynamic coordination with no doubt will increase productivity to a higher level because here, the use of emotions is minimized. Considering an example: A and B belong to two different communities who are in conflict with each other and work at the same association/organization which results in quite a hostile relationship between the two and might affect their productivity and mental state. While normal coordination here might help them work together to an average level, dynamic coordination with no doubt will be increasing their proficiency as here, they will be putting aside their hostile emotions because of external factors and will be focusing on work with the primary objective being productivity. Dynamic coordination is a long process and cannot be achieved without proper training. 


\section{Implications of Terrestrial Variations on the utility of Theory of Special Neurodiversity}

And for this training to be successful, people need to make efforts and be invested in it because here what we need is a change of mindset through mutual equilibrium. To quote it in the words of James P.

Leynse, 'You can't clap with one hand'.

\section{CONCLUSION}

The present study was carried out in the view of geographical variations for the utilization of the 'Theory of Special Neurodiversity'. For a vast country like India, the poor management of this diversity and these variations can act as a hindrance in the path of national growth and development. For the proper optimization of the key parameters, a survey was conducted along with addressing the role of geography in the utility 'Theory of Special Neurodiversity'. Based on the results, in view of much needed socioeconomic growth following conclusions may be drawn:

1) Theory of Special Neurodiversity along with 'RAS-VIN's model' when utilized as a whole can result in the accelerated socioeconomic growth of the nation.

2) Geography by no doubt plays a significant role in the optimization of the 'Theory of Special Neurodiversity'. Furthermore, change is dynamic in nature and unavoidable.

3) The three key parameters i.e., culture, climate, and coordination were explored. It can be concluded that it is impossible to make this theory work with singularity because each of these parameters has its significant role or position and impact on each other. Therefore, the solutions need to be in a coupled or unified form.

4) A distinct proportion of these three factors through a new novel model i.e., the 'Dynamic Coordination Equilibrium Model' is proposed which at any geographical location, throughout the world can help in the maximum utilization of the theory of special neurodiversity.

5) The coordination needs to be dynamic and not normal. Furthermore, the coordination needs to be mutual, or else, equilibrium won't be maintained.

6) Proper optimization of the 'Dynamic Coordination Equilibrium Model' in respect to the 'Theory of Special Neurodiversity' will result in adequate growth of an individual with special needs as a person along with enabling them to be a significant contribution to the development of the nation as a whole.

7) Applications of the work: Present study and insights can be effectively utilized into wide range of organizational, practical, functional, institutional, engineering, scientific research, technological applications.

\section{ACKNOWLEDGMENT}

The authors acknowledge the contribution of all the survey participants who made this research possible. We further dedicate this work to people with special needs working hard for the development of the nation.

\section{REFERENCES}

1) Hall, E., 'The entangled geographies of social exclusion/inclusion for people with learning disabilities', Health \& Place 11(1): 107-115,1995.

2) Park, D. C., Radford, J. P., and Vickers, M. H., 'Disability studies in human geography', Progress in Human Geography 22,2, pp. 208-233, 1998.

3) Lemon, C., and Lemon, J., 'Community based cooperative ventures for adults with intellectual disabilities', Canadian Geographer, 47(4):414 - 428, 2003.

4) Imrie, R., and Edwards, C., 'The Geographies of Disability: Reflections on the Development of a Sub-Discipline', Geography Compass 1/3: 623-640, 2007.

5) Singal, N., 'Forgotten Youth: Disability and Development in India', VIII ${ }^{\text {th }}$ International Sociological Association Conference on 'Asian Youth and Childhoods' Lucknow, India, Nov. 22- 24, 2007.

6) Appunni, S. S., and Deshpande, A. P., 'Work Participation among Disabilities in India', Social Sciences, 21(2): 117-122, 2009.

7) Shenoy, M., 'Persons with disability \& the India labour market: Challenges and opportunities', ILO (International Labour Organization) Asia-Pacific working paper series, 2011.

8) Kumar, S. G., Roy, G., and Kar, S. S., 'Disability and Rehabilitation Services in India: Issues and Challenges', Journal of Family Medicine and Primary Care, Volume 1: Issue 1, 2012.

9) Jindal, A. R., and Chari, R., 'Employment scenario of people with disabilities in India:20 Years of The Disability Act, 1995', National Centre for Promotion of Employment for Disabled People (NCPEDP), 2015.

10) Limaye, S., 'Factors Influencing the Accessibility of Education for Children with Disabilities in India', Global Education Review, 3 (3). 43-56, 2016. 


\section{Implications of Terrestrial Variations on the utility of Theory of Special Neurodiversity}

11) Mehta, M., Kumari, R., Sharma, K., and Chandani, A., 'Are Indian Organizations Disabled Friendly? - Inclusive Talent Acquisition Function for Indian Organizations', International Journal of Innovative Technology and Exploring Engineering (IJITEE), Volume-8 Issue-12S2, 2019.

12) Seilan, A., 'Prevalence and Distribution of Disability in India', The Hindecon, Issue:22, Pages 3339, 2020.

13) Malhotra, R., and Malhotra, V., 'Theory of Special Neurodiversity: Implications and Applications', Proceedings of Academics World International Conference, New Delhi, India, March-2021. Attribution - Non Commercial 4.0 International (CC BY-NC 4.0)

(https://creativecommons.org/licenses/by-nc/4.0/), which permits remixing, adapting and building upon the work for non-commercial use, provided the original work is properly cited. 